\title{
A Denoising Algorithm for Complex Surface Image based on Adaptive Gaussian Filter Model
}

\author{
Lizhong Tu, Jiande Zhang, Qing Yang and Yan Zhuang \\ School of Computer Engineering, Nanjing Institute of Technology, Nanjing 211167, China; \\ zhangjiandexy@163.com
}

Keywords: Gaussian Filter, Adaptive filter, Image denoising, Complex surface.

\begin{abstract}
This paper presents an adaptive Gaussian filter model to solve complex curve surface image denosing problem. Based on the considering of local distribution characteristics of image noise, an adaptive Gaussian filter model is constructed by introduced an automatic adjusting smooth scale parameter, which is calculated by the distance of the gray value of the current pixel and the average gray value of all pixel in the window. Experimental results show that the adaptive denosing strategy is able to remove noise and preserve details of complex curve surface. The researched model is superior to conventional Gaussian filter model.
\end{abstract}

\section{Introduction}

Complex curve surface images collected from real environment usually have a lot of uneven distribution noise. How to remove noise and improve image quality is always a focus of digital image process technology[1-3]. Based on distribution of image spectrum, traditional image denoising algorithms distinguish useful information from noise by smoothing technology[4-6], and high frequency parts of image will be removed. As some useful information are also in the high frequency parts, edges of image usually become blurred and some image details are lost during the process of removing noise. Considering global and local characteristics of image and researching an adaptive image denoising algorithm are of great significance.

An image denoising algorithm based on adaptive Gaussian filter model is researched in this paper. Take the distance between the gray value of current pixel and the average gray value of all pixel in the window as a smooth scale parameter, an adaptive Gaussian filter model is constructed and applied to image denoising. By the new algorithm, global characteristics as well as local characteristics of image are used to calculate model parameters.

Principles of adaptive Gaussian filter model are described in Section 2. Section 3 presents the implementation process of the algorithm in details. An application to real image width the researched algorithm is carried out in Section 4, and the results are shown to verify our method. Concluding remarks give an account in Section 5.

\section{Principle of adaptive Gaussian filter}

Complex curve surface images tend to have obvious uneven characteristics. Referring to the general algorithm [7-8], it is difficult to remove noise from complex curve surface image efficiently because parameters in the model cannot be calculated out in the process of image denoising. We can construct a new model to solve the denoising problem of complex curve surface image, the process is described as figure 1 .

Based on the analysis of typical complex curve surface images, noises which are difficult to deal are usually on the edge of target in image. When Gaussian filter model is applied to denoising, we expect a bigger smooth scale parameter for noise pixels while a smaller for edge pixels, because noise pixels are discrete while edge pixels are continuous [9-11]. So, the smooth scale parameter is the most relevant and important parameter for denoising model of complex curve surface. In order to consider the local characteristics of image, introduced an automatic adjusting smooth scale parameter 
calculated by the distance of the gray value of the current pixel and the average gray value of all pixel in the window to the new model is a good choice. Then, complex curve surface image can be denoised by the new adaptive model.

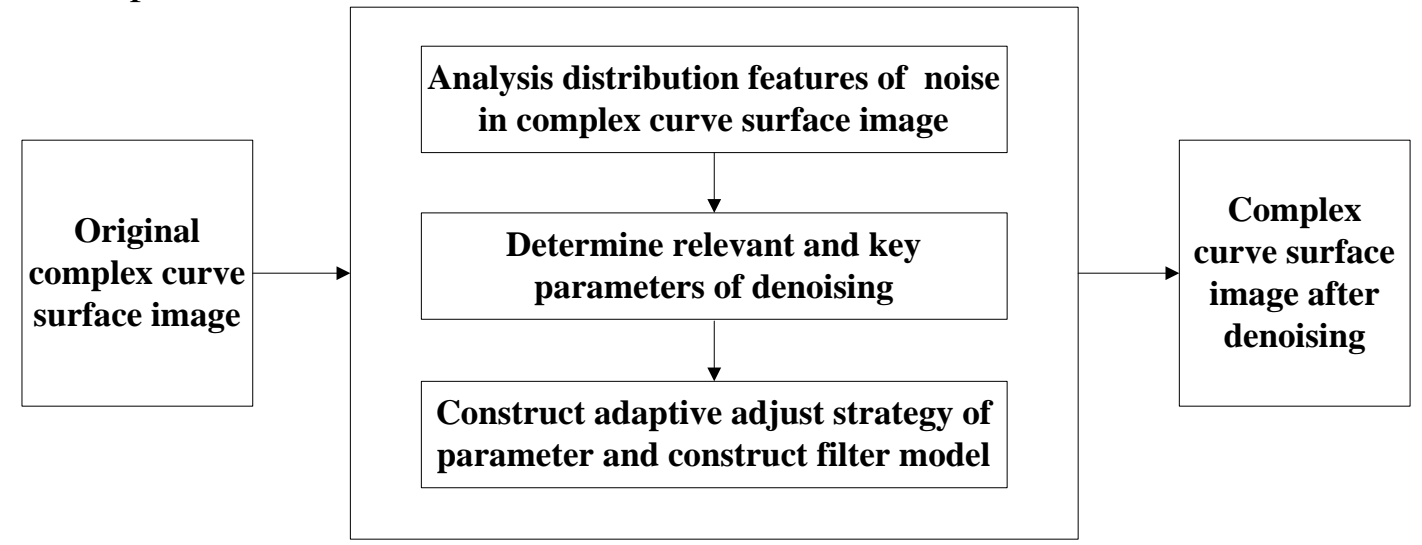

Fig 1. Principle of image adaptive denoising

\section{Computer implementation of adaptive Gaussian filter}

The new model discussed above can be applied to the complex curve surface image denoising problem. The procedure is shown as figure 2 and can be described as fellows:

Step 1. Read the original complex curve surface image and save the information of each pixel to construct an image space. $\mathrm{M}^{*} \mathrm{~N}$ is the size of the Gaussian filter, to ensure the reading in not out-or boundry, value intervals for $\mathrm{M}$ and $\mathrm{N}$ are $[M, l$ Width $-M]$ and $[N, l$ Height $-N]$, respectively;

Step 2. Determine the size of the filter template. To do denoising effectively, it is not suitable to choose a too bigger template. We choose the filter window with a size $3 * 3$ (that is $\mathrm{M}=3, \mathrm{~N}=3$ ) ;

Step 3. Calculate grey distance of current pixel. Supposed that $f(x, y)$ is the gray value of the point $(x, y), l D i s t$ is the absolute value of grey difference for the current pixel and the average value of all pixel's grey values in neighborhood. The grey distance can be calculated out below:

$$
\begin{aligned}
& \text { lDist }=\mid f(x, y)-\frac{1}{9}[f(x-1, y-1)+f(x, y-1)+f(x+1, y-1)+f(x-1, y)+ \\
& f(x, y)+f(x+1, y)+f(x-1, y-1)+f(x, y-1)+f(x+1, y-1)] \mid
\end{aligned}
$$

Step 4. Determine Gaussian filter for current pixel. The smooth scale parameter is the grey distance calculated out in step 3 and the adaptive Gaussian filter constructed in this paper can be described as:

$$
G(x, y)=\frac{1}{\sqrt{2 \pi} \sigma} e^{-\frac{x^{2}+y^{2}}{2 \sigma^{2}}}
$$

Where, $g$ is the image, $g(x, y)$ is the gray value of one pixel point in image $g, h$ is the height of image, $\mathrm{w}$ is the width of image ;

Step 6. Based on the Gaussian filter determined above, the complex curve surface image can be denoised finally. 


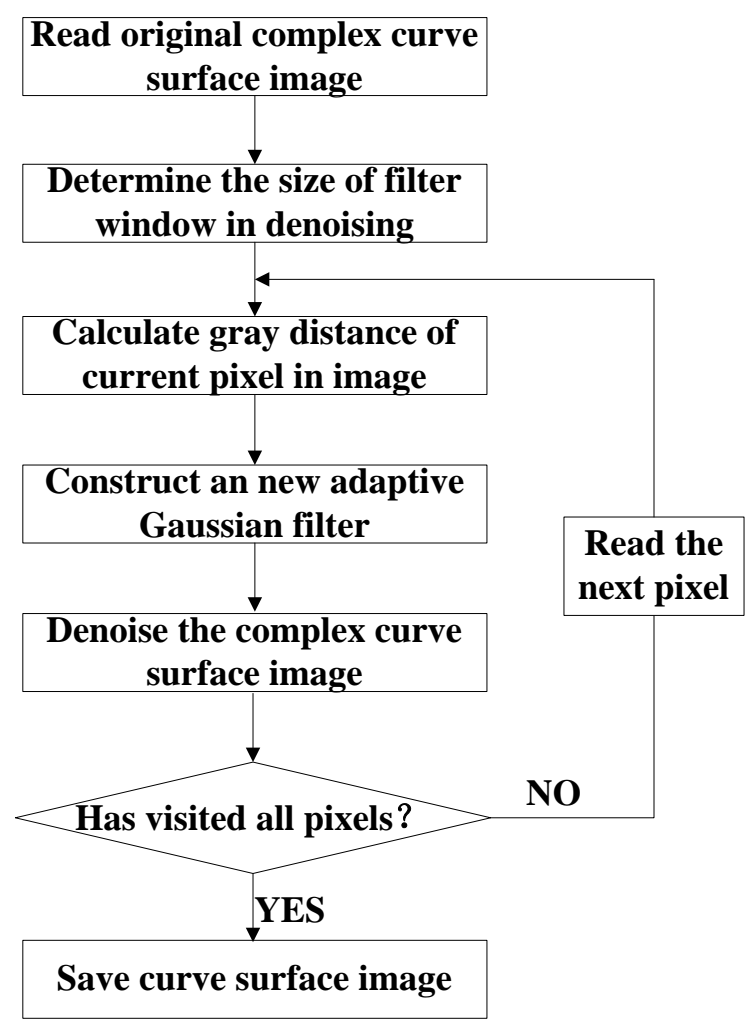

Fig 2. Flowchart of image adaptive denoising

\section{Numerical Experiments}

Experiments are taken out to verify the electiveness of researched algorithm by applying it to a complex curve surface image. The original image is collected by HiVision Dcam 100M(industrial camera) and Computar M1214(camera lens). The original image is shown in figure 3, and the denoised image is shown in figure 4. The result of experiment is shown as follow:

From the experiment result, we can see that: in the original image, there are lots of noises near the edge of blade and the edge is not very obvious, the contrast in the image is low. While, when the image has been denoised by the algorithm presented in this paper, we obtain a satisfactory images which has been improved very well, and the complex curve surface is well balanced.
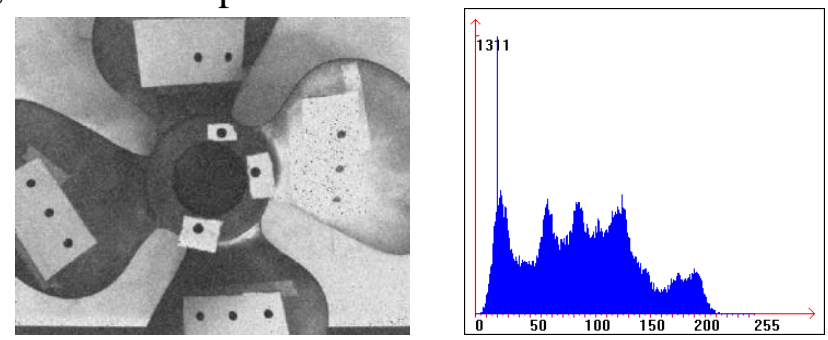

Fig 3. Original image and its histogram
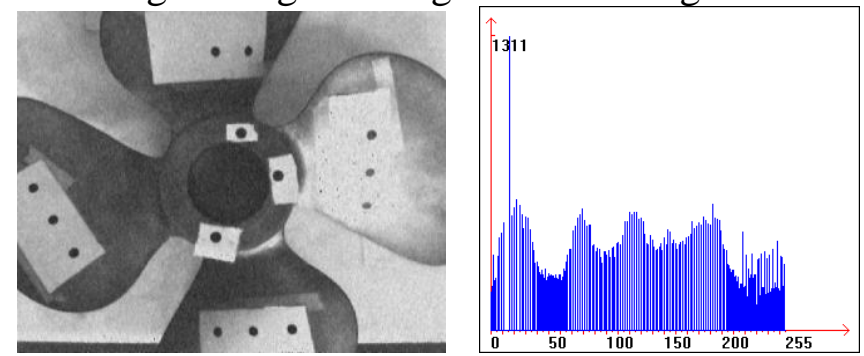

Fig 4. Denoised image and its histogram 


\section{Conclusion}

The adaptive image denoising strategy researched in this paper can choose suitable Gaussian filter automatically by local information of image. Experimental results of complex surface surface image indicate the validity of the research work. The image denoised prepares a good foundation for image segmentation.

\section{Acknowledgements}

This work is supported in part by the Natural Scientific Research Foundation of JiangSu Province (Grant No.BK20150731), Natural Scientific Research Foundation of Colleges in JiangSu Province (Grant No.15KJB520014) and Scientific Research Foundation of Nanjing Institute of Technology (Grant No.CKJA201306). We are grateful to express our thanks.

\section{References}

[1]. Yusuke Tokuyoshi. Modified filtered importance sampling for virtual spherical Gaussian lights. Computational Visual Media. Vol.27 (2016) No.4, p.343-355.

[2]. Yonggang Zhang,Yulong Huang. Gaussian approximate filter for stochastic dynamic systems with randomly delayed measurements and colored measurement noises. Science China (Information Sciences). Vol.59 (2016) No.9, p.165-182.

[3]. Abdulkadir Eryildirim,Mehmet B. Guldogan. A Gaussian mixture Bernoulli filter for extended target tracking with application to an ultra-wideband localization system. Digital Signal Processing. Vol.5 (2016)No.7, p.1-12.

[4]. H.H. Afshari, S.A. Gadsden,S. Habibi. Gaussian Filters for Parameter and State Estimation: A General Review of Theory and Recent Trends. Signal Processing. Vol.13 (2017) No.5, p.1-12.

[5]. Yali Wang,Brahim Chaib-draa. KNN-Based Kalman Filter: An Efficient and Non-stationary Method for Gaussian Process Regression. Knowledge-Based Systems. Vol.11 (2016)No.4, p.148-155.

[6]. Wenlong Huang,Xiaodan Wang,Guohong Li. Immune adaptive Gaussian mixture particle filter for state estimation. Journal of Systems Engineering and Electronics. Vol.26 (2015) No.4, p.877-885.

[7]. B. Liu,M.E. Gharamti,I. Hoteit. Assessing clustering strategies for Gaussian mixture filtering a subsurface contaminant model. Journal of Hydrology. Vol.535 (2016)No.3, p.1-21.

[8]. C. Combastel. An Extended Zonotopic and Gaussian Kalman Filter (EZGKF) merging set-membership and stochastic paradigms: toward non-linear filtering and fault detection. Annual Reviews in Control.Vol.4 (2016) No.2, p.232-243.

[9]. Liu Yu,Dong Kai,Wang Haipeng, et al. Adaptive Gaussian sum squared-root cubature Kalman filter with split-merge scheme for state estimation. Chinese Journal of Aeronautics. Vol.27 (2014)No.5, p. 1242-1250.

[10]. Sheng Jin,Dianhai Wang,Cheng Xu,et al. Short-term traffic safety forecasting using Gaussian mixture model and Kalman filter. Journal of Zhejiang University-Science A(Applied Physics \& Engineering). Vol.14 (2013) No.4, p.231-243.

[11]. Junyi Zuo, Yingna Jia. Simplified unscented particle filter for nonlinear non-Gaussian Bayesian estimation. Journal of Systems Engineering and Electronics.Vol.24 (2013) No.3, p.537-544. 